\title{
The physical meaning of a nonlinear evolution equation of the fourth order relating to locally and non-locally supercritical waves
}

\author{
A. Huber ${ }^{1,2}$ \\ ${ }^{1}$ Address constantly: Prottesweg 2a, A-8062 Kumberg, Austria \\ ${ }^{2}$ Institute of Theoretical Physics - Computational Physics, Technical University Graz, Petersgasse 16, A-8010 Graz, Austria \\ E-mail: soliton.alf@web.de
}

\begin{abstract}
In this paper the physical meaning of a nonlinear partial differential equation (nPDE) of the fourth order relating to wave theory is deduced to the first time. The equation under consideration belongs to a class of less studied nPDEs and an explicit physical meaning is not known until now. This paper however bridges the gap between some known results and a concrete application concerning wave theory. We show how one can derive locally supercritical solitary waves as well as locally and nonlocally forced supercritical waves and analytical solutions are given explicitly.
\end{abstract}

Keywords: Nonlinear partial differential equations, evolution equations, supercritical solitary waves, locally supercritical waves, non-locally supercritical waves.

\section{Introduction - outline of the problem - some known results}

The scaled nPDE e.g. (Huber, 2008a,b) in $(1+1)$ dimension under consideration is given by:

$$
\frac{\partial^{2} u}{\partial t^{2}}+\frac{\partial^{4} u}{\partial^{3} x \partial t}+3 \frac{\partial^{2} u}{\partial x^{2}} \frac{\partial^{2} u}{\partial x \partial t}=0, u=u(x, t), u \in C^{4}(-\infty, \infty), t>0,
$$

where the function $u(x, t)$ describes a wave propagation depending on time $t$. We seek for classes of solutions for which $u=F(x, t)$, where $F \in C^{4}(D)$ and $D \subset R^{2}$. Hint: In what follows, we suppress the item 'classes', so 'classes of solutions' are simply 'solutions'.

In recent papers (Huber, 2005, 2008a), the tanh-approach was used to calculate soliton-solutions of the nPDE, eq.(1). Alternatively, new solutions can also found in (Huber, 2005, 2008a,b) and (Huber, 2007).

It is known that there exist two different types of single soliton-solutions; regular solutions as well as irregular solutions which have singularities where the arguments of the traveling waves vanish.

Further, the author has proven the Painlevé-conjecture (Huber, 2008a), so we conclude that the nPDE, eq.(1) possesses the Painlevé-property. It was shown that general solutions of eq.(1) are expressed in terms of elliptic functions by use of the Weierstrassian expansion method (Huber, 2008a) and moreover, the application of the classical Lie group formalism also leads to new types of solutions (Huber, 2008a).

Although several solutions of eq.(1) exist no direct connection to a physical problems is known. Now, the purpose is to present a concrete application of physical relevance to the first time.

We start by using a frame of reference introducing $u(x, t)=f(\xi)$ with $\xi=x-\lambda t, \lambda=$ const. into eq.(1) to derive the nODE

$$
\frac{d^{4} f}{d \xi^{4}}+3\left(\frac{d^{2} f}{d \xi^{2}}\right)^{2}-\lambda \frac{d^{2} f}{d \xi^{2}}=0
$$


Putting $h=f^{(2)}$ as new dependent variable we have the following nODE of the second order:

$$
\frac{d^{2} h}{d \xi^{2}}+3 h^{2}-\lambda h=0
$$

In context to soliton theory $\lambda$ means the soliton's velocity and we point out that there are four types of solutions possible:

Case A: Supercritical solitary waves: They occur only for $\lambda>0$ and sufficiently large,

Case B: Supercritical stationary cnoidal waves: They occur only for $\lambda<0$ and sufficiently small,

Case C: Stationary hydraulic fall: Only if $\lambda$ admits a special value, so that $\lambda_{L}<0$,

Case D: Unsteady periodic soliton radiation: This case appears if $|\lambda|$ is small (sometimes known as transcritical solutions).

\section{Supercritical solitary waves}

Here we assume eq.(1.2) in the following general form with suitable boundary conditions (BC):

$$
\beta h^{\prime \prime}+\alpha h^{2}-\lambda h=g(\xi),-\infty<\xi<\infty, \lambda>0, h^{\prime}(\xi= \pm \infty)=h(\xi= \pm \infty)=0,
$$

and the prime means derivation w.r.t the independent variable $\xi$.

$g(\xi)$ is a given function (the forcing) which is differentiable and has a compact support (i.e. it is nonzero only in a closedbounded set). Recall that $(1+\varepsilon \lambda) \sqrt{g H}$ is the upstream near the critical flow speed and in our case, $\alpha=3$ and $\beta=1$ holds. Physical intuition tells that for a given $\alpha, \beta$ and $g(\xi)$ the shape of the free surface is controlled by the upstream flow velocity, e.g. $\lambda$, (Benjamin, 1967; Benjamin and Feir, 1967 and (Camassa and Wu, 1991). Therefore, it is of interest to study the solution behavior for different values of $\lambda$.

The forcing function $g(\xi)$ is due to the bottom topography of a fluid domain or due to an external pressure on the free water surface. We shall see that there exist a number $\lambda_{C}>0$ so that eq.(1) has (i) at least two solutions for $\lambda>\lambda_{C}$, (ii) one solution for $\lambda=\lambda_{C}$ and (iii) no solution if $\lambda<\lambda_{C},($ Craik, 1985) and (Coles, 1965).

\subsection{Locally forced supercritical waves}

In scaling processes, especially those of an ideal flow over a small bump, (Hammack and Segur, 1974; Gurtin, 1975; Grimshaw, 1987) the height $\|\sigma\|$ of the bump is divided by $H$ and the length of the bump base $B$ should be divided by the horizontal length scale $L$.

If the length is very short then $B / L$ is very small since $L$ is very large for long wave assumption. After scaling we regard the base length of the bump as zero.

But the area under the bump is not zero. It is known that Dirac's function possesses such a property. The forcing that has a very short base length is called the local forcing. So we consider for the nODE eq.(2):

$$
\beta h^{\prime \prime}+\alpha h^{2}-\lambda h=\frac{P}{2} \delta(\xi), \lambda>0,
$$

looking for solitary wave solutions of eq.(2.1). Hence h satisfies the BC:

$$
h^{\prime}(\xi= \pm \infty)=h(\xi= \pm \infty)=0 \text {. }
$$

By direct integration solution of eq.(2.1) connected by the BC (2.2) are given explicitly in terms of hyperbolic secant functions

$$
\begin{aligned}
& h(\xi)=-\frac{3 \lambda}{2 \alpha} \operatorname{sech}^{2}\left[\sqrt{\frac{-\lambda}{4 \beta}}\right]\left(\xi-C^{+}\right), \quad \xi>0 \\
& h(\xi)=-\frac{3 \lambda}{2 \alpha} \operatorname{sech}^{2}\left[\sqrt{\frac{-\lambda}{4 \beta}}\right]\left(\xi-C^{-}\right), \quad \xi<0,
\end{aligned}
$$

where $\mathrm{C}^{+}$and $\mathrm{C}^{-}$are constants. The continuity condition for the free surface at $\xi=0$ gives $h\left(0^{+}\right)=h\left(0^{-}\right) \equiv h(0)$ implying that $C^{+}= \pm C^{-}$holds. Recalling the property of $\delta(\xi)$, that means that $h^{\prime}(\xi)$ must have a jump discontinuity at $\xi=0$, so that

$$
h\left(0^{+}\right)-h^{\prime}\left(0^{-}\right)=\frac{P}{2 \beta} \text {, or with } \beta=1 \text { it follows that } h\left(0^{+}\right)-h^{\prime}\left(0^{-}\right)=\frac{P}{2} \text { holds. }
$$

This condition can be rewritten by considering eq.(2.3) to give the expression 


$$
-\sqrt{\frac{-\lambda}{\beta}} h(0)\left[\operatorname{tg}\left(\sqrt{\frac{-\lambda}{4 \beta}} C^{+}\right)+\operatorname{tgh}\left(\sqrt{\frac{-\lambda}{4 \beta}} C^{-}\right)\right]=\frac{P}{2 \beta} .
$$

Eq.(2.5) holds for a nonzero $\mathrm{P}$ only if $C^{+} \neq C^{-}$and from $C^{+}= \pm C^{-}$we have $C^{+}=-C^{-}=C_{0}$. Hence, eq.(2.5) can be written as

$$
\frac{d^{3} f}{d f^{3}}-f-c=0 \text {, where } f=\operatorname{tgh}\left(\sqrt{\frac{-\lambda}{4 \beta}} C_{0}\right) \text { and } c=\frac{P \alpha}{6 \lambda} \frac{1}{\sqrt{-\beta \lambda}}, f=f(\xi) .
$$

Since we can write $f^{3}-f=f(f+1)(f-1)$ in polynomial form it follows that we have three possibilities once again:

(i) when $|c|<2 / 3 \sqrt{3}$, eq.(2.6) has three distinctive roots,

(ii) when $|c|>2 / 3 \sqrt{3}$, eq.(2.6) has only one real root with the absolute value $>1$,

(iii) when $|\mathrm{c}|=2 / 3 \sqrt{3}$, eq.(2.6) has a double root.

Therefore the constant $\mathrm{c}$ becomes

$$
c=-\frac{P \alpha}{6 \lambda} \frac{1}{\sqrt{-\beta \lambda}}= \pm \frac{2}{2 \sqrt{3}}
$$

and determines the critical value of $\lambda$ so that a closed-form expression is obtained:

$$
\lambda_{C}=\sqrt[3]{\frac{3 \alpha^{2} P^{2}}{-16 \beta}}
$$

Moreover a concrete expression for the constant $C_{0}$ follows immediately by

$$
C_{0}=\sqrt{\frac{4 \beta}{-\lambda}} \operatorname{arctgh}(f) \text {. }
$$

So we have three possibilities once again: (i) $C_{0}$ has two values $\lambda>\lambda_{C}$, (ii) only one value for the choice $\lambda=\lambda_{C}$ and (iii) there exists no solution in case of $0 \leq \lambda \leq \lambda_{C}$.

For a given $C_{0}$ the solutions of eq.(2.3) are determined to give the following hyperbolic functions once again

$$
h(\xi)=-\frac{3 \lambda}{2 \alpha} \begin{cases}\operatorname{sech}^{2} \sqrt{\frac{-\lambda}{4 \beta}}\left(\xi-C_{0}\right), & \text { for } \xi \geq 0 \\ \operatorname{sech}^{2} \sqrt{\frac{-\lambda}{4 \beta}}\left(\xi+C_{0}\right), & \text { for } \xi \geq 0\end{cases}
$$

If a special choice for the constant $C_{0}$ is made, (2.6d) defines cusped solitary waves (Huber, 2008c). The cusp is concave up (down) if $C_{0}>0,\left(C_{0}<0\right)$ respectively.

From the equations (2.6), (2.6a) and (2.6c) we can assume the following chain of relations:

$$
\begin{aligned}
& P<0 \rightarrow c<0 \rightarrow f>0 \rightarrow C_{0}>0, \text { that means the cusp is concave up, } \\
& P>0 \rightarrow c>0 \rightarrow f<0 \rightarrow C_{0}<0, \text { that means the cusp is concave down. }
\end{aligned}
$$

Otherwise from (2.6e) one can assume the relation: $\operatorname{sign}(P)=-\operatorname{sign}\left(C_{0}\right)$.

Further from (2.6d) and (2.6e) which determines the free surface profile, we conclude that if $P<0,(P>0)$, then the cusps of the solitary waves are concave up (down).

That means that a surface suction $(P<0)$ corresponds to a dent of the free surface and a surface pressure $(P>0)$ correlates to a crest of the free surface.

Remark: In the case of $P<0$ we have $\|h\|_{\infty}=-(3 \lambda) /(2 \alpha) \operatorname{sech}^{2} \sqrt{-\lambda /(4 \beta)}<-(3 \lambda) /(2 \alpha)$, that is the amplitude of the free solitary wave. The bifurcations diagram $\|h\|_{\infty}$ vers. $\lambda$ is given by 


$$
\|h\|_{\infty}=-\frac{P}{4} \sqrt{\frac{-3}{\beta \lambda}}\left\{\cos \left[\frac{1}{3} \arccos \left(-\frac{P \alpha}{6 \sqrt{-\beta \lambda^{2}}}\right)+\left\{\begin{array}{l}
4 \pi / 3 \\
2 \pi / 3
\end{array}\right]\right\}^{-1} .\right.
$$

The curve of $\left(\|h\|_{\infty}, \lambda\right)$ has two branches in principal. The upper (lower) branch correlates to $4 \pi / 3,(2 \pi / 3)$, respectively.

These two branches are joined at $\lambda_{C}$ on which $\left\|h_{1}\right\|_{\infty}=-\lambda_{C} / \alpha$. As $P \rightarrow 0^{+}, C_{0,1} \rightarrow 0^{-}$and $C_{0,2} \rightarrow \infty$. Hence $\|h\|_{\infty}$ approaches to $-3 \lambda / 2 \alpha$ and zero, respectively.

For $P<0$, the amplitude $\|h\|_{\infty}$ is equal to $-3 \lambda / 2 \alpha$ all the time. The cusp solitary waves have two peak in each single solution and as $P \rightarrow 0^{-}$the two peaks disappear gradually.

\subsection{Non-locally forced supercritical waves}

The non-local forcings are special classes and cannot be approximated by a delta function. In general, such problems have to be solved numerically (especially BVP and IVP). Referring to eq.(2) we solve this equation in the domain $\left(-\infty, \xi_{-}\right]$analytically.

An analytic solution of eq.(2), considering the above given domain, is given by

$$
h(\xi)=-\frac{3 \lambda}{2 \alpha} \operatorname{sech}^{2}\left[\sqrt{\frac{-\lambda}{2 \beta}}\right]\left(\xi-C_{0}\right), \quad \xi<\xi_{-},
$$

where we assume the constant $C_{0}$ as a pure phase shift. If we consider the appearance of a bump, say $g(x)$ in general as above, the constant $C_{0}$ cannot be chosen arbitrarily. It is known that $C_{0}$ can only take up some discrete values; the next step naturally is to determine these values (Whitham, 1974; Miles, 1986).

Therefore we introduce a new quantity $\mathrm{E}_{\lambda}\left(\mathrm{C}_{0}\right)$ defined through

$$
E_{\lambda}\left(C_{0}\right):=\int_{\xi_{-}}^{\xi_{+}} g(\xi) h^{\prime}(\xi) d \xi
$$

or similarly

$$
E_{\lambda}\left(C_{0}\right)=\frac{\beta}{2}\left\{h^{\prime}\left(\xi_{+}\right)\right\}^{2}+\left[\frac{\lambda}{2}+\frac{\alpha}{3} h\left(\xi_{+}\right)\right] h^{2}\left(\xi_{+}\right) .
$$

Assuming a half solitary wave (e.g. $\left.\xi>\xi_{+}, h(+\infty)=0\right)$ the polynomial $Q(\xi)$, defined by

$$
Q(h)=E_{\lambda}\left(C_{0}\right)-\left(\frac{\lambda}{2}+\frac{\alpha}{3}\right) h^{2},
$$

must therefore have a double zero smaller than the third real zero. This happen if $E_{\lambda}\left(C_{0}\right)=0$ and we conclude that the following relation holds: $h(+\infty)=0 \Leftrightarrow E_{\lambda}\left(C_{0}\right)=0$.

If $E_{\lambda}\left(C_{0}\right)$ is valid, then the function $h(\xi)$ satisfies $0<h(\xi)<-3 \lambda /(2 \alpha)$ for $\xi>0$. Hence, $E_{\lambda}\left(C_{0}\right)=0$ is the condition to determine $C_{0}$. In general $C_{0}$ is an implicit function of $\lambda$ which may be multiple valued. To derive $C_{0}$ we solve the IVP up to $\xi_{+}$similarly to eq.(2):

$$
\beta h^{\prime \prime}+\alpha h^{2}-\lambda h=0, \quad \xi>\xi_{-},
$$

with

$$
\begin{aligned}
& h\left(\xi_{-}\right)=-\frac{3 \lambda}{2 \alpha} \operatorname{sech}^{2}\left[\sqrt{\frac{-\lambda}{4 \beta}}\right]\left(\xi_{-}-C_{0}\right), \\
& h^{\prime}\left(\xi_{-}\right)=-\sqrt{\frac{-\lambda}{\beta}} h\left(\xi_{-}\right) \operatorname{tgh}\left[\sqrt{\frac{-\lambda}{4 \beta}}\right]\left(\xi_{-}-C_{0}\right) .
\end{aligned}
$$

Usually, starting by a trial value of $C_{0}$ the above given initial conditions could derived. The given IVP eq.(2.10a) and eq.(2.10b) have a unique solution since the nODE, eq.(2.10) satisfies the Lipschitz condition. So we can plot the function $E_{\lambda}$ vs. $C_{0}$ for a fixed $\lambda$. 
Finally we give some hints to relevant papers, especially relating to supercritical phenomena. These citations cover several applications in civil engineering and related domains:

Supercritical flow interaction occurring in the marine boundary layer between closely spaced coastal capes discusses (Haacks and Burk, 2001) and surface waves related with roughness is found in Toombes and Chanson (2007). The case of supercritical flow at weirs is studied in (Ghodsian, 2003); a simulation of the supercritical flow by using the classical shallow water equations gives Krüger and Rütschmann (2006) as well as Tjernström and Grisogno (2000). A general introduction is suggested in Subramania (1997).

\section{Analysis, results and discussion}

Before we discuss the main problem, eq.(2.1) let us start by computing some solutions of eq.(1.2). Introducing a new variable so that $p(h(\xi))=h^{\prime}(\xi)$ we derive the following nODE of the first order:

$$
p p^{\prime}+3 h^{2}-\lambda h=0, p=p(h), p \neq 0 \text {. }
$$

This equation admits solutions expressed as $p(h)= \pm \sqrt{h^{2}-2 h^{3}-c_{1}}$ where $c_{1}$ is an arbitrary constant of integration (it is sufficient to consider only the upper sign of the root).

Integrating once again we derive at the function $h(\xi)$ by $\int \frac{d h}{p(h)}=\left(\xi-\xi_{0}\right)$, where $\xi_{0}$ is a further constant of integration. Several special cases are considered:

(i) $\lambda=1$ and $c_{1}=0$ : this elementary case allows a solution by

$$
h(\xi)=\frac{1}{2}\left[\left\{\operatorname{ctgh}^{2}\left(\frac{1}{2}\left(\xi-\xi_{0}\right)-1\right)\right\}\left\{\operatorname{tgh}^{2}\left(\frac{1}{2}\left(\xi-\xi_{0}\right)\right)\right\}\right]=\frac{1}{1+\cosh \left(\xi-\xi_{0}\right)}, \xi \neq \pm \mathrm{i} \pi .
$$

Typical solitary solutions occur; in Figure 1 we show the waves for different values of the constant $\xi_{0}$.
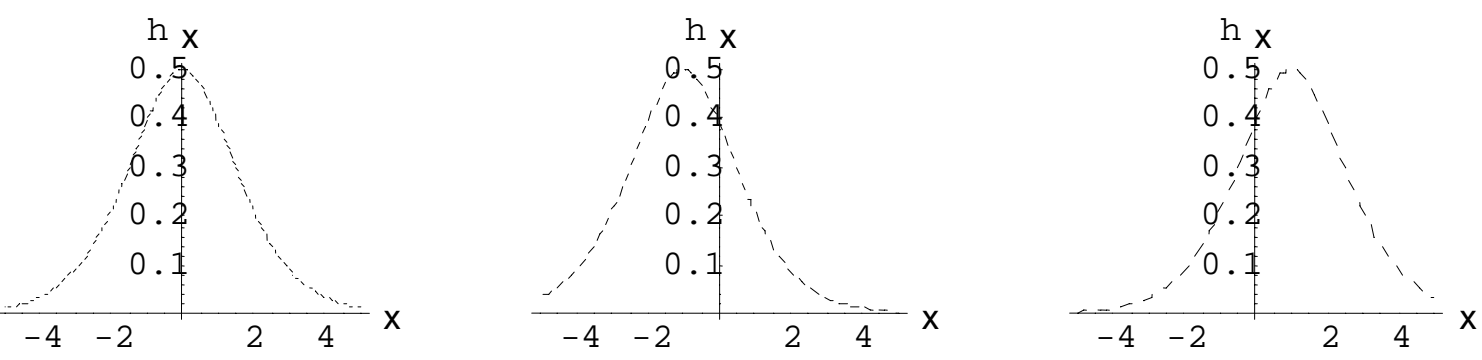

Figure 1 Typical pure solitary solutions of the eq.(3) for different values of the phase shift constant $\xi_{0}$.

Acting as a phase shift the constant $\xi_{0}$ influences the wave so that the crest twists right or left; on the other hand by vanishing constant the peak is symmetrically to the vertical axis.

To be a solitary wave the function has to be vanishing for $\xi \rightarrow \pm \infty$ and in fact this condition is fulfilled and for $\xi \rightarrow 0 \pm$ the solution tends to a finite value. (ii) the general case with $\lambda=c_{1}=$ arbitrary can be handled only by numerical standard methods leading to an elliptic integral of the first kind. For the case (iii) $\lambda=1$ and $c_{1}=-1$, we calculate:

$$
h_{1,2}(\xi)=\frac{i}{4}\left[i-\sqrt{7}+2 \sqrt{7} s^{2}\left\{ \pm \sqrt{\frac{(5+i \sqrt{7}) \xi_{0}-(5+i \sqrt{7}) \xi}{2 \sqrt{2}}}\right\}, \frac{2 \sqrt{7}}{\sqrt{7}-5 i}\right], \quad i^{2}=-1 \text {, }
$$

$\xi_{0}$ means an arbitrary integration constant and numerically the complex-valued modulus is $m=0,4375+0,8268 i$.

These solutions can be regarded as a further new contribution to existing solutions. Both the Figure 2 and Figure 3 represent the contour graphics as well as mappings onto the complex plane, respectively. Two poles can be explicitly seen together with the distribution of the field lines around them (for simplicity we assume the constant $\xi_{0}$ to be vanishing and consider the plus sign). 

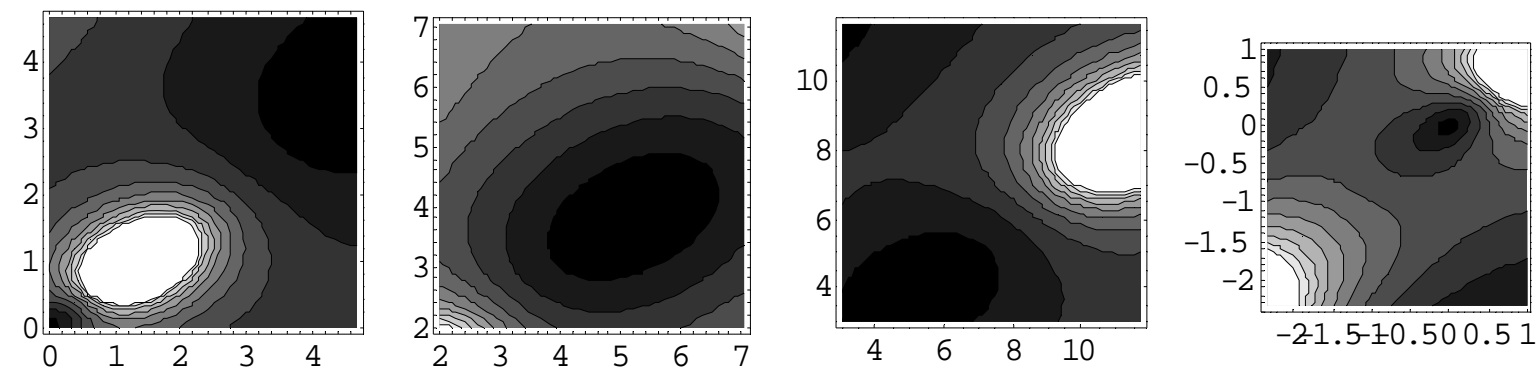

Figure 2 Contour charts of the solution (3.2) for different values of the complete elliptic integral $K(k)$ in the complex plane, we assume the integration constant $\xi_{0}=0$. Two poles are shown surrounded by their branch lines.
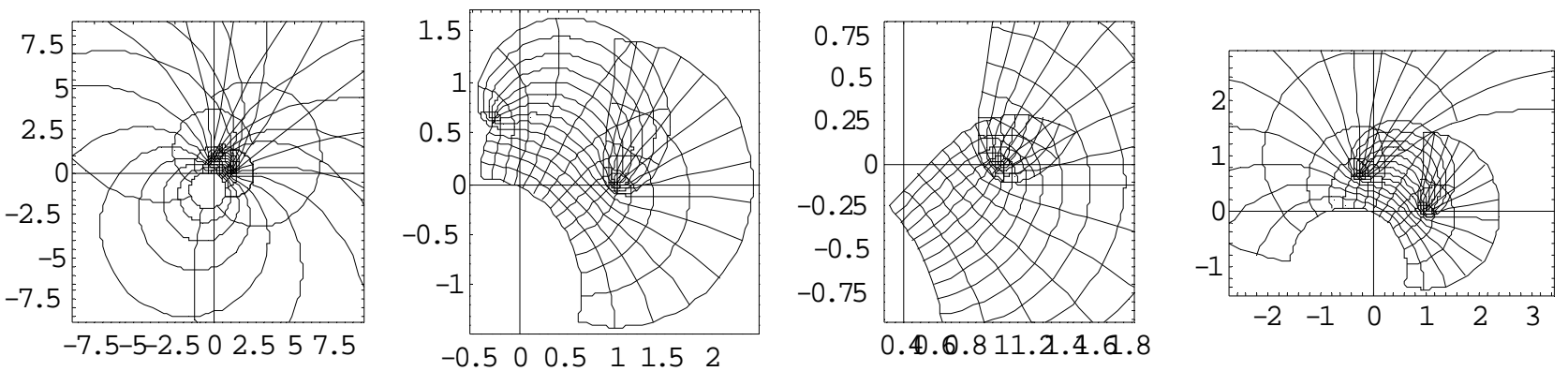

Figure 3 Altitude charts of the solution (3.2) for different values of the domain: from left to right: $0<\xi<2 \pi, 0<\xi<\pi$, $0<\xi<\pi / 2$ and $0<\xi<3 \pi / 2$. In the first and third domain it seems that the poles move together.

Let us now introduce some special cases for the modulus of the solution (3.2), especially $k=0$ and $k=1$, respectively to derive the sine and their hyperbolic varieties.

Splitting up these limiting cases into a real and a complex part we calculate for the real parts:

$$
\begin{aligned}
& \operatorname{Re}\left[h_{1}(\xi)\right]=-\frac{\sqrt{7}}{4} \sin [\sqrt[4]{8} \sqrt{\xi}] \times \sinh [\sqrt[4]{8} \sqrt{\xi}] \text { for the case } m=0 \\
& \operatorname{Re}\left[h_{1}(\xi)\right]=-\frac{1}{4}-\frac{\sqrt{7} \sin [\sqrt[4]{8} \sqrt{\xi}] \times \sinh [\sqrt[4]{8} \sqrt{\xi}]}{(\cos [\sqrt[4]{8} \sqrt{\xi}]+\cosh [\sqrt[4]{8} \sqrt{\xi}])^{2}} \text { for the case } m=1
\end{aligned}
$$

The function (3.2a) vanishes in the limit as $|\xi| \rightarrow 0$ and takes infinite as $|\xi| \rightarrow \infty$. The limiting behavior for (3.2b) is similar to (3.2a) in case of $|\xi| \rightarrow \infty$ but the function takes a finite value as $|\xi| \rightarrow 0$.

Both of the real parts can be seen in Figure 4. The first and the second derivatives of the real parts are discontinuously differentiable at the point $\xi=0$ and have singularities there.
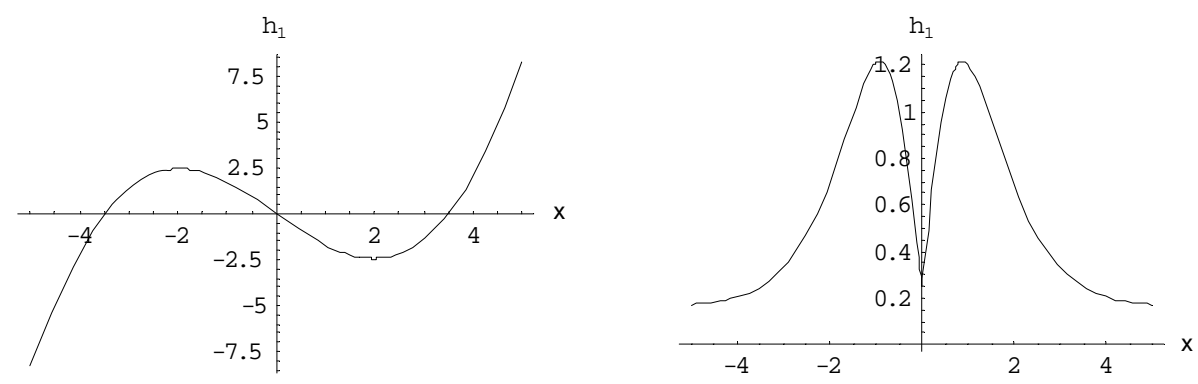

Figure 4 Planar plots of the real parts of the functions eq.(3.2a) right and eq.(3.2b) left. A remarkable discontinuity on the vertical axis is observed, the peaks are symmetrically regarding also to the vertical axis. 
Asymptotically for example, for large values of the argument, that is $|\xi| \rightarrow \infty$, we have for the function (3.2.a) the series representation up to order four (and similar for eq.(3.2.b)):

$$
\operatorname{Re}\left[h_{1}(\xi)\right]=-\frac{1}{4}-\sqrt{\frac{7}{2}}\left(\frac{1}{\xi}\right)+\frac{17 \sqrt{14}}{45}\left(\frac{1}{\xi}\right)^{3}+O\left[\left(\frac{1}{\xi}\right)\right]^{4} .
$$

Some notes relating to the interpretation in the Poincaré $(p, h)$-phase plane of eq.(3) may not be omitted. The critical points $P_{1}(0,0)$ and $P_{2}(0,1 / 3)$, respectively are unstable nodes.

To see this it is shown that the eigenvalues at $P_{1}$ are $\lambda= \pm i$; so the origin is an unstable node and for $P_{2}$ we have $\lambda= \pm 1$; therefore an unstable saddle point. Unstable hence the limit for $p(h)$ tends to $-\mathrm{i} \infty$ as $h \rightarrow \pm \infty$ in case of $\lambda>0, c_{1}>0$ and $\lambda<0, c_{1}<0$. In Table 1 we summarize the complete limiting behavior and Figure 5 represents characteristic curves in the phase plane.

\begin{tabular}{||c|c|c|c||}
\hline Case & Choice of the parameters & $\lim _{h \rightarrow \pm 0} p(h)$ & $\lim _{h \rightarrow \pm \infty} p(h)$ \\
\hline 1 & $\lambda>0, c_{1}>0$ & -1 & $-\mathrm{i} \infty$ \\
\hline 2 & $\lambda<0, c_{1}<0$ & $-\mathrm{i}$ & $-\mathrm{i} \infty$ \\
\hline 3 & $\lambda>0, c_{1}<0$ & $-\mathrm{i}$ & $-\mathrm{i} \infty$ \\
\hline 4 & $\lambda<0, c_{1}>0$ & -1 & $-\mathrm{i} \infty$ \\
\hline
\end{tabular}

Table 1. Limiting cases of the function $p(h)$, eq.(3) in the Poincaré $(p, h)$-phase plane, $i^{2}=-1, c_{1}$ is a constant, $\lambda$ the wave number.
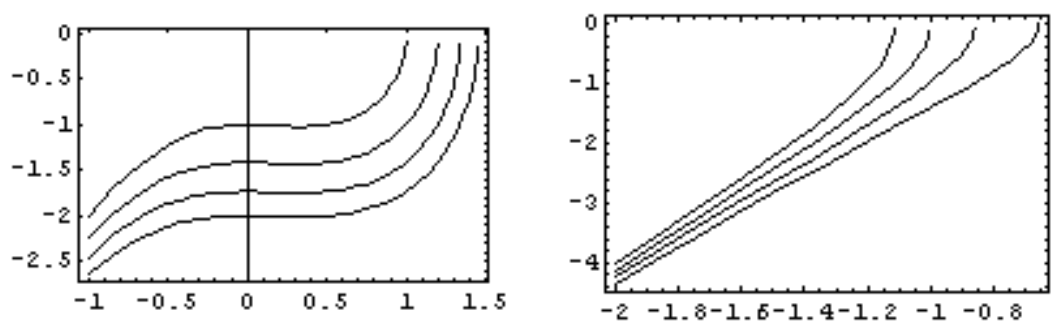

Figure 5 Phase plane trajectories $p=p(h)$ for the nODE eq.(3), left: positive values for $c_{1}$, right: negative values of the constant $c_{1}$ are used. Note: The singular points are not considered.

The non-locally supercritical function eq. (2.8) possesses all necessary properties to be a soliton, i.e. the function rapidly vanishes as $|\xi| \rightarrow \pm \infty$ but it is remarkable that the solution becomes unstable. Figure 6 shows the analytical solution eq.(2.8) resulting in a typical supercritical solitary motion. 

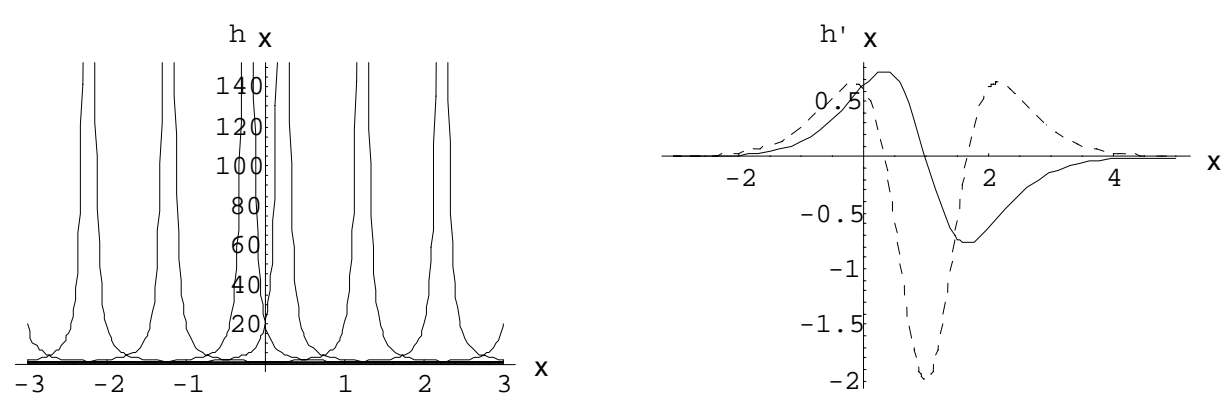

Figure 6 Left: Typical supercritical solitary motion generated by the eq.(2.8) with $\lambda=-2$ and $c_{1}=1$. Right: the first derivative (full line) and the second derivative (dotted line) with the same parameter choice.

Let us now discuss the locally forced supercritical waves, especially eq.(2.1) with a small change so that we can consider $\beta h^{\prime \prime}+\alpha h^{2}-\lambda h=\frac{P}{2} \delta(\xi)$ but $\lambda<1$ and $h(\xi=-\infty)=h^{\prime}(\xi=-\infty)=0$. When $\xi<0$ the solution vanishes identically, that is in detail $h(\xi) \equiv 0$ as $\xi \leq 0$.

Therefore solving this problem given above is equivalent to solve the IVP: $\beta h^{\prime \prime}+\alpha h^{2}-\lambda h=0, h\left(0^{+}\right)=0$, $h^{\prime}\left(0^{+}\right)=P / 2 \beta$.

So, the relating quantity $E_{\lambda}\left(C_{0}\right)$, eq.(2.9a) as a first integral together with the polynomial of the third order in our case admits:

$$
E_{\lambda}\left(C_{0}\right)=\frac{1}{2} h^{\prime 2}=-\left[\frac{\lambda}{2}+h\right] h^{2}, \quad Q(h)=E_{\lambda}-\left(\frac{\lambda}{2}+h\right) h^{2}, \quad \xi>0, h\left(0^{+}\right)=0
$$

So we can plot the polynomial $Q(h)$ vs. the function $h$. Moreover the dependence $E_{\lambda}\left(C_{0}\right)$ vs. $C_{0}$ for a fixed $\lambda$ is seen in Figure 7.

We remark that the polynomial (3.3) must have a double root which is, indeed fulfilled (independently of the value of $\lambda$, e.g. $\lambda<0$ and/or $\lambda>0$ we have $h_{1,2}=0, h_{3}=-1 / 2$ ).
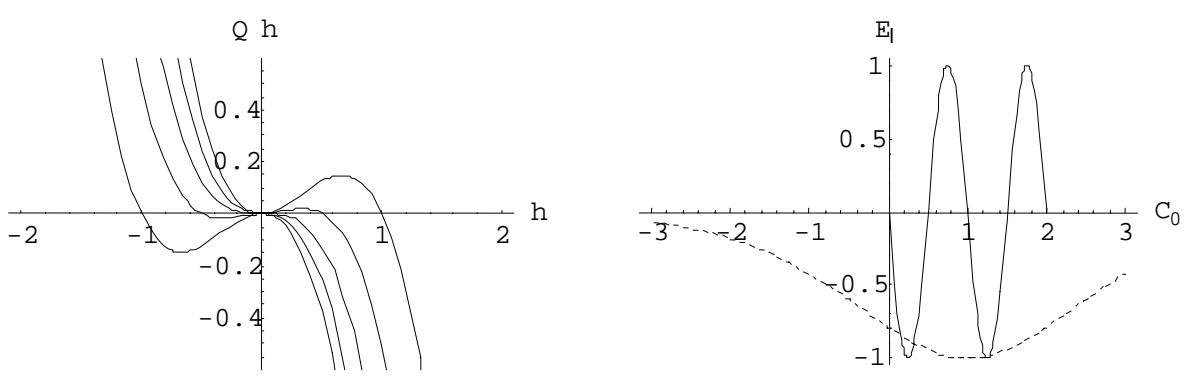

Figure 7 Left: Graphical plot of the polynomial of the third order, eq. (3.3), right: The connection $E_{\lambda}\left(C_{0}\right)$ vs. $C_{0}$ for a fixed $\lambda$.

\section{Summary}

The present paper deals with an nPDE of the forth order. The crucial aspect however is the fact that up to now no direct physical application is known. We show to the first time the relation to wave propagation concerning supercritical waves by considering a special force term, the Dirac's delta function. By applying the procedure another remarkable result could derive: It is shown how that the nPDE under consideration admits new types of solutions in terms of Jacobian functions. The cases of locally and nonlocally forced supercritical waves (including solitary-like) are studied in detail. Some remarks relating the behavior in the phase plane are given. So the present paper is suitable to expand the knowledge of the nPDE under consideration. Finally, to clarify the analysis, some important graphic simulations are presented. 


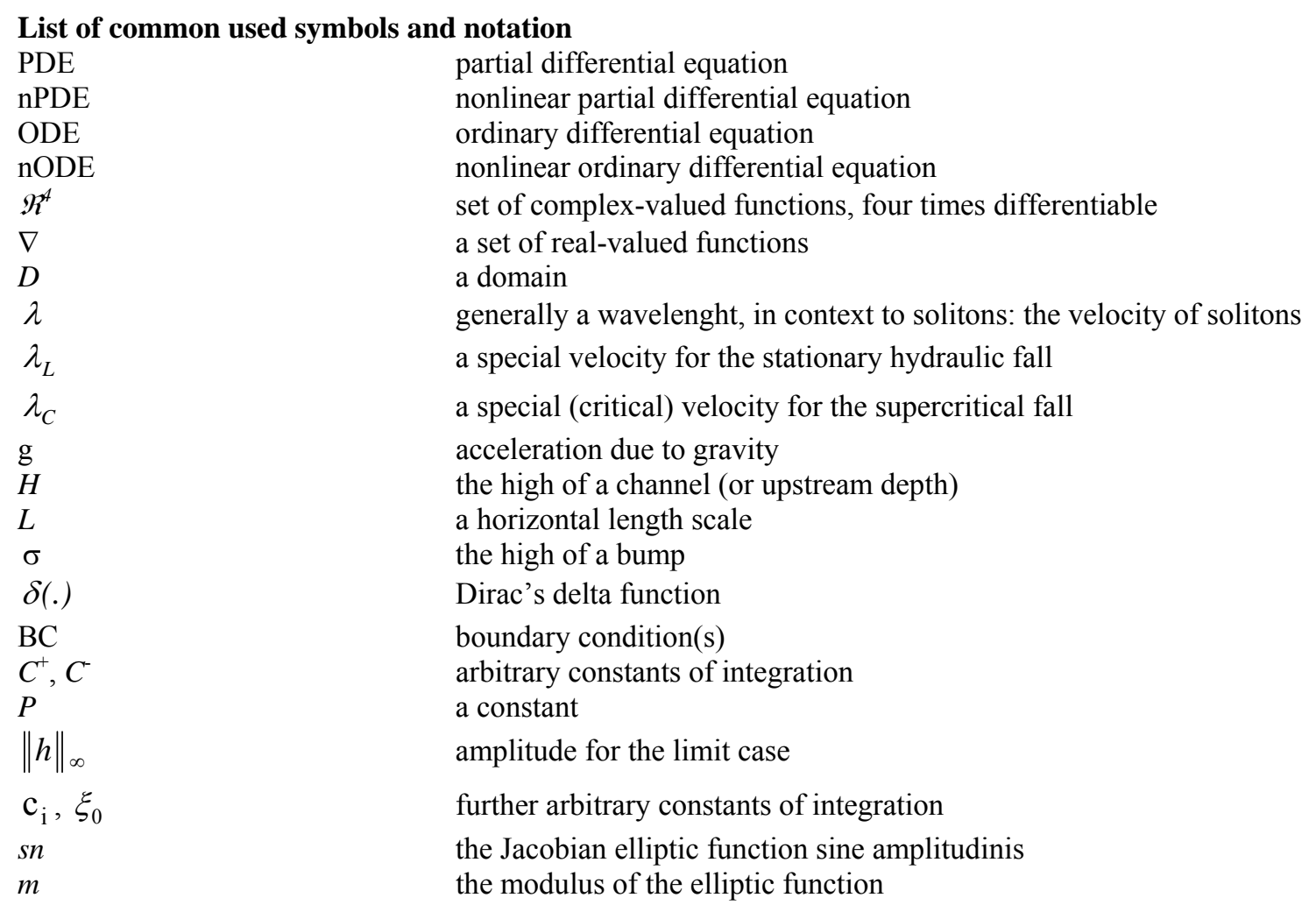

\section{Acknowledgements}

The author would like to dedicate the present paper to the much honoured Univ.-Prof. Dipl.-Phys. Dr. rer. nat. Wolfgang von der Linden, Head of the Institute of Theoretical Physics - Computational Physics at the Technical University of Graz, Austria. The author is further grateful to Professor Dr. Wolfgang von der Linden for kindly supporting his scientific intensions.

\section{References}

Benjamin T.B., 1967. Instability of periodic wave trains in nonlinear dispersive systems, Proc. Roy. Soc. London A, Vol. 229 , p.59. Benjamin T.B., Feir J.F., 1967. The desintegration of wave trains on deep water, Part 1, Theory, J. Fluid. Mech. Vol. 27 , p.417.

Camassa R., Wu T.Y., 1991. Stability of forced solitary waves, Trans. R. Roy. London A Vol. 337, p.429.

Craik A.D.D., 1985. Wave interaction and Fluid Flows, University Press, New York.

Coles D., 1965. Transition in circular Cuette flow, J. Fluid. Mech. Vol. 21, p.385.

Ghodsian M., 2003. Supercritical flow over a rectangular side weir, Can. J. of Civil Eng. Vol. 30, No. 3, p.596.

Grimshaw R., 1987. Resonant forcing of barotropic coastally trapped waves, J. Phys. Oceanagr. Vol. 17, p.53.

Gurtin M.E., 1975. On the breaking of water waves in a sloping beach of arbitrary shape, Q. Appl. Math. Vol. 33, p.187.

Hammack J.L., Segur H., 1974. The Korteweg-de Vries equation and water waves, Part 2, Comparison with experiments, J. Fluid. Mech. Vol. 65, p.289.

Haacks T., Burk S.D., 2001. Supercritical flow interaction within the Cape Blanco - Cape Mendocino orographic complex, Amer. Meterological Soc. Vol. 129, No. 4, p.688.

Huber A., 2008a. The calculation of novel class of solutions of a non-linear fourth order evolution equation by the Weierstraß transform method, Appl. Math. and Comp. Vol. 201, p.668.

Huber A., 2008b. A note on new solitary and similarity class of solutions of a fourth order non-linear evolution equation, Appl. Math. and Comp., Vol. 202, p.787.

Huber, A., 2008c. A note on a class of solitary-like solutions of the Tzitzéica-equation generated by a similarity reduction, Physica D Vol. 237, p.1079.

Huber A., 2005. Solitary solutions of some nonlinear evolution equations, Appl. Math. and Comp. Vol. 166, No. 2, p.464.

Huber A., 2007. A novel algebraic procedure for solving non-linear evolution equations of higher order, Chaos, Solitons and Fractals, Vol. 34, No. 3, p.765.

Krüger S., P. Rütschmann P., 2006. Modelling 3D supercritical flow with extended shallow-water approach, J. Hydraulic Eng. Vol. 132, No. 9, p.916 
Miles J.W., 1986. Stationary transcritical channel flow, J. Fluid. Mech. Vol. 162, p.489.

Subramania K., 1997. Flow in Open Channels, McGraw Hill.

Tjernström M., Grisogno B.B., 2000. Simulation of supercritical flow around points and capes in a costal atmosphere, $J$. Atmospheric Sci. Vol. 57, No. 1, p.108.

Toombes L., Chanson H., 2007. Surface waves and roughness in self-aerated supercritical flow, Environmental Fluid. Mech. Vol. 7, No. 3, p.259.

Whitham G.B., 1974. Linear and Nonlinear Waves, J. Wiley, New York.

\section{Biographical notes}

Dipl.-Ing. Dr. techn. Huber Alfred is a distinguished lecturer at the Institute of Theoretical Physics - Computational Physics at the Technical University Graz, Austria following his habilitation treatise. He did his diploma thesis titled 'Systematic in the physics of elementary particles focusing the quarkonium states' in the field of elementary particle physics at the former Institute of Nuclear Physics at the Technical University Graz, Austria. He completed his scientific education with the doctoral programme of technical sciences at the Institute of Chemical Technology of Inorganic Compounds at the Technical University Graz, Austria subject to nuclear solid state physics and advanced electrochemistry. Thesis titled 'Synthesis and characterization of doped $\gamma$-manganese dioxides'. Also the author has a learnt vocation for a chemical assistant at the Research Centre of Electron Microscopy at the former Technical High School Graz, Austria. He is the author of 27 articles which have appeared in world-wide renowned scientific journals. His research interests are nonlinear partial differential equations (nPDE) of higher order with applications especially in physics and chemistry. The author developed several new algebraic procedures for solving nPDE. Special interests are further given in classical and non-classical symmetry methods, nonlinear transformations and the application of nonlinear methods in describing electrochemical interfaces, nonlinear wave propagation and further nonlinear topics of advanced character.

Received December 2009

Accepted December 2009

Final acceptance in revised form January 2010 